\title{
Elastic Formation Control Based on Affine Transformations
}

\author{
Lara Briñón Arranz, Alexandre Seuret and Carlos Canudas de Wit
}

\begin{abstract}
This paper deals with the control of a fleet of nonlinear systems representing AUVs (autonomous underwater vehicles). The purpose is here to design a control law to stabilize the fleet to time-varying formations which are not only circular. A novel framework is proposed to express a general control law for a large class of formations. This is produced by applying a sequence of affine transformations such as translations, rotations and scalings. The paper also includes a cooperative control to distribute the agents along the formation which takes into account the communication constraints. The system was implemented in computer simulation, accessible through Web ${ }^{1}$.
\end{abstract}

\section{INTRODUCTION}

Cooperative control problems and multi-agent systems have received much attention in recent years. The field includes consensus algorithms for multi-agent systems [1], [2], flocking [3], distributed sensor networks [4], [5], [6], trajectory tracking and path following [7], [8] and autonomous systems as underwater and unmanned air vehicles (AUVs and UAVs) [9], [10]. Formation control and motion coordination have been extensively studied, see [11], [12], among many others. Control laws have been provided to make a fleet of agents (vehicles) obtain circular and parallel formations [4], [13]. Many extensions based on these works have been developed: three-dimensional formation control [14], planar circular formation control in a flow-field [15], translation [16] and scaling [17] of circular formations, and stabilization of a fleet to other closed forms [18], [19].

The objective of this paper is to design a general control law for a class of non-linear multi-agent systems to reach many class of formations including non-circular and timevarying formations. Based on [4], new results have been already proposed in [16] and [17] to deal with time-varying formations resulting from translating and contracting a circular formation. Nevertheless, generalization of these ideas to the called here elastic formation is pertinent to some applications where the agents should perform collaborative tasks requiring the formation to move towards an a priori unknown direction and to adapt to some particular form. For instance, in source seeking applications, the formation should displace in the source gradient direction and contract its size to adapt to the level curves of the source plume, [20].

Translation, scaling and rotation are the three fundamental transformations of a formation [21]. These three

Lara Briñón Arranz is with INRIA Rhône-Alpes, NeCS Team, Grenoble, France lara.brinon-arranz@inrialpes.fr

Alexandre Seuret and Carlos Canudas de Wit are with the Department of Automatic Control, GIPSA-Lab, CNRS, NeCS Team, Grenoble, France \{alexandre.seuret, carlos.canudas-de-wit\} @ipsa-lab.grenoble-inp.fr

This work was supported by EU STREP project FeedNetBack FP7-ICT2007-2.

${ }^{1}$ Simulations are accessible in the CONNECT project web at http://www.lag.ensieg.inpg.fr/connect/. main affine transformations, usually used in the fields of geometric and robotic control, are pertinent to express many class of closed curves in a matrix representation. A closed curve can be expressed as a sequence of transformations applied to the unit circle. In [18], a general framework based on affine transformations is presented. This idea allows to control the agents to different trajectories which results from the application of these three affine transformations. The contribution of this paper is to stabilize the agents to the same elastic formation, not only to the same velocity reference, independently of initial conditions. A time-varying closed curve defined by a sequence of affine transformations is considered as an elastic formation. Our approach considers that this closed curve is know for all the agents (i.e. the sequence of transformations is a given reference).

In the context of the source seeking for underwater vehicles, it is relevant to constrain the agents in an appropriate shape to avoid unnecessary energy waste. Moreover, ensuring that the agents are uniformly distributed along the formation might be more adequate to produce efficient search motions, [20]. Therefore, an additional component of the control law is also added to distribute of the agents along the elastic formation. This is achieved by taking into account the communication graph between the agents. The collaborative control law stands for the case of rangedependent graph.

The following section presents the problem formulation introducing the affine transformation and the model of the agents. Section III exposes the main contribution of the article which deals with the control law to stabilize the agents to an elastic formation and a cooperative control law to distribute the agents along the formation. Section V presents some particular closed formations and the simulation results.

\section{PROBLEM FORMULATION AND PRELIMINARIES}

\section{A. Model of agents and Problem Formulation}

Consider the standard agent model commonly used in the literature to model AUVs restricted kinematics [4], [13], [19]. It corresponds to a kinematic unicycle fitting with model properties subject to a simple non-holonomic constraint, adequate for the underwater vehicles. Consider a set of $N$ agents, in which each agent $k=1, \ldots, N$ has the following constrained dynamics:

$$
\begin{aligned}
\dot{x}_{k} & =v_{k} \cos \theta_{k} \\
\dot{y}_{k} & =v_{k} \sin \theta_{k} \\
\dot{\theta}_{k} & =u_{k}
\end{aligned}
$$

where $\left(x_{k}, y_{k}\right)^{T}$ is the position vector of agent $k, \theta_{k}$ is the heading angle and $v_{k}, u_{k}$ are the control inputs. 
Whit appropriate limits on the control inputs, this model can provide a reasonable approximation for many air and underwater vehicles.

The objective is to stabilize the fleet of agents into a richer class of formations, i.e. non-circular and time-varying formations. In [4], a complex notation was introduced to formulate in an simple manner the circular formation control law using the previous kinematic model with unit velocity $v_{k}=1 \forall k$. Here, the vectorial notation (1) is now employed and a simple formulation of the control law is obtained.

Moreover, an additional objective is to distribute the agents along the formation in a cooperative way, taking into account the communication constraints.

\section{B. Preliminaries on Affine Transformations}

A circular formation in the plane can be defined by three basic parameters, the center of the circle, its radius and the angular velocity of the agents along the circle. In order to modify these parameters, the affine transformations are introduced.

The three main transformations are the translation, the rotation and the scaling. To express these affine transformations the homogeneous coordinates are defined, [22]. The homogeneous coordinates of a vector $\mathbf{z} \in \mathbb{R}^{2}$ can simply be defined as the new vector $\mathbf{z}^{h}=\left(z_{x}, z_{y}, 1\right)^{T}$. Let the vectors $e_{1}=(1,0,0)^{T}, e_{2}=(0,1,0)^{T}, e_{3}=(0,0,1)^{T}$ be a canonic base of the space $\mathbb{R}^{2}$ expressed in homogeneous coordinates. In the sequel, the basic affine transformations and some of their properties are presented.

a) Translation: The translation $\mathcal{T}$ of a point $\mathbf{z}$ by a vector c corresponds to the following operation $\mathcal{T}(\mathbf{z})=$ $\mathbf{z}+\mathbf{c}$. This can be expressed in a matrix multiplication of the form $\mathbf{z}^{\prime}=\mathbf{T}_{c} \mathbf{z}^{h}$ where

$$
\mathbf{T}_{c}=\left(\begin{array}{ccc}
1 & 0 & c_{x} \\
0 & 1 & c_{y} \\
0 & 0 & 1
\end{array}\right)
$$

and $\mathbf{z}^{\prime}$ is expressed in homogeneous coordinates. Its inverse exists and satisfies $\mathbf{T}_{c}^{-1}=\mathbf{T}_{-c}$. Note that $\mathbf{c}$ can be timevarying. This translation is pertinent to move the center of the formation, see Fig. 1.

b) Scaling: A non-uniform scaling expressed in homogeneous coordinates is a transformation such that $\mathbf{z}^{\prime}=\mathbf{S} \mathbf{z}^{h}$ where

$$
\mathbf{S}=\left(\begin{array}{ccc}
s_{x} & 0 & 0 \\
0 & s_{y} & 0 \\
0 & 0 & 1
\end{array}\right)
$$

and $s_{x}>0, s_{y}>0$. Its inverse matrix contains the inverse of its elements. The parameters of the scaling can be timevarying. Some examples of scalings can even lead to ellipses or other closed curves as shown in Fig. 1.

c) Rotation: A rotation through an angle $\alpha$ counterclockwise around the origin can be written in a matrix form as previously $\mathbf{z}^{\prime}=\mathbf{R}_{\alpha} \mathbf{z}^{h}$ where

$$
\mathbf{R}_{\alpha}=\left(\begin{array}{ccc}
\cos \alpha & -\sin \alpha & 0 \\
\sin \alpha & \cos \alpha & 0 \\
0 & 0 & 1
\end{array}\right)
$$

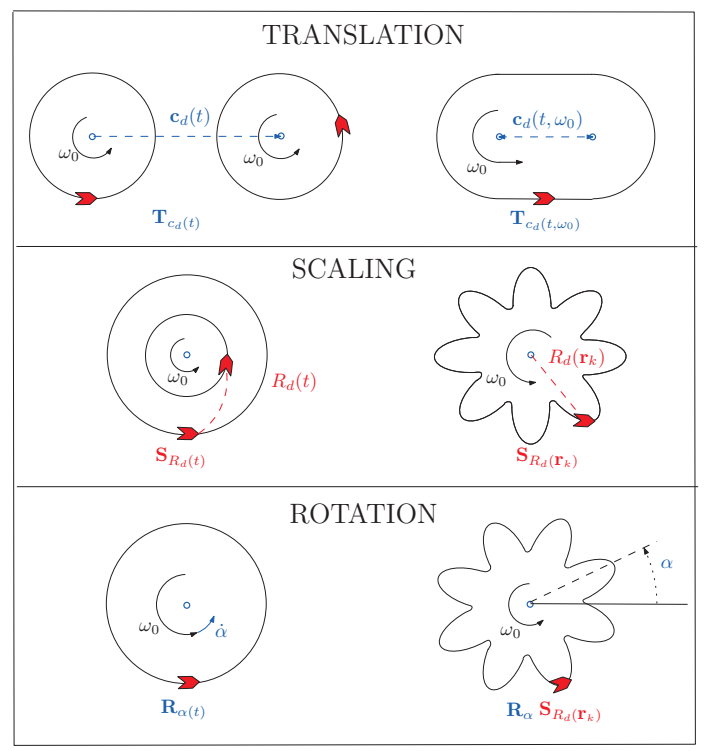

Fig. 1. Affine transformations applied to the circular formation

Its inverse exists and satisfies $\mathbf{R}_{\alpha}^{-1}=\mathbf{R}_{\alpha}^{T}$. The angle $\alpha$ can be time-varying. A rotation applied to a formation can change its orientation with respect to the frame origin or, in the case of a circular formation, it affects the angular velocity of the agents around the circle as shown in Fig. 1.

The objective is to find a general framework to stabilize the fleet of agents to an elastic formation using these affine transformations. An elastic formation is defined as a closed curve which results of applying a sequence of affine transformations to the unit circle (i.e. a circle centered at the origin with unit radius). This elastic formation can be time-varying if at least one element of the transformation matrices is time-varying. In the sequel, a sequence of affine transformations, which are generated by a combination of the previous ones, is defined as follows:

$$
\mathbf{G}=\prod_{i}^{I} \prod_{j}^{J} \prod_{k}^{K} \mathbf{S}_{i} \mathbf{R}_{\alpha_{j}} \mathbf{T}_{c_{k}}
$$

where the subscripts denote the different transformations of the same type which are applied. For instance, the matrix $\mathbf{G}=\mathbf{S}_{1} \mathbf{S}_{2} \mathbf{R}_{\alpha} \mathbf{T}_{c}$ is a combination of one translation, one rotation and two different scalings. Note that the matrix multiplication is not commutative. However, the general transformation $\mathbf{G}$ considered here, is a sequence of the three affine transformations and the order defined in (2) can be changed, for instance, to $\mathbf{G}=\mathbf{R}_{\alpha} \mathbf{S}_{1} \mathbf{T}_{c} \mathbf{S}_{2}$, which defines an other elastic formation.

As it is shown in the previous paragraph, the affine transformations are invertible, therefore the inverse matrix of the general transformation $\mathbf{G}^{-1}$ exists. Thanks to previous definitions, $\mathbf{G}$ and $\mathbf{G}^{-1}$ are differentiable, if their parameters are differentiable. Note that the operators derivative and invertible are not commutative, therefore $\left(\frac{d}{d t} \mathbf{G}\right)^{-1} \neq$ $\frac{d}{d t}\left(\mathbf{G}^{-1}\right)=\dot{\mathbf{G}}^{-1}$.

In this paper a general transformation is applied to the unit circle to provide different non-circular and time-varying 
formations. The final formation depends on the sequence used to define G. In other words, starting from a circle, a large class of formations can be obtained.

\section{Control Design}

In previous section, the basic affine transformations have been defined. The objective is now to design a control law such that the fleet of agents reach an elastic formation defined by any sequence of affine transformations applied to the unit circle.

\section{A. Control Design: Change of Coordinates}

Following the previous section, the problem becomes a formation control design based on the circular control law from [4]. The desired elastic formation is defined by applying the matrix $\mathbf{G}$ to unit circle $\mathcal{C}_{0}$. The stabilization of system (1) to an elastic formation can be expressed as the stabilization of an adequate transformed system to the unit circle.

The position vector of the agent $k$ in homogeneous coordinates is defined as $\mathbf{r}_{k}=\left(x_{k}, y_{k}, 1\right)^{T}$. The main contribution of this paper is to introduce the coordinates transformation:

$$
\hat{\mathbf{r}}_{k}=\mathbf{G}^{-1} \mathbf{r}_{k}
$$

where $\hat{\mathbf{r}}_{k}=\left(\hat{x}_{k}, \hat{y}_{k}, 1\right)^{T}$ is the transformed position vector expressed in homogeneous coordinates. The main idea is first, to stabilize this new transformed system to a circle with unit radius $R_{0}=1$, centered at $(0,0)$ and with angular velocity $\omega_{0} \neq 0$. Then, to apply the circular control law from [4]. Finally, to apply the inverse transformation to express the control law in the original framework.

In order to apply the circular formation control law, the new transformed system must have constant linear velocity equal to $R_{0} \omega_{0}$. Therefore the dynamics of the transformed position vector are defined as:

$$
\begin{aligned}
& \dot{\hat{x}}_{k}=\omega_{0} \cos \psi_{k} \\
& \dot{\hat{y}}_{k}=\omega_{0} \sin \psi_{k}
\end{aligned}
$$

where $\psi_{k}$ represents the angular orientation of the transformed velocity vector $\dot{\hat{\mathbf{r}}}_{k}$ such that:

$$
\psi_{k}=\arctan \frac{e_{2}^{T} \dot{\hat{\mathbf{r}}}_{k}}{e_{1}^{T} \dot{\hat{\mathbf{r}}}_{k}}+\epsilon \pi=\arctan \frac{e_{2}^{T} \frac{d}{d t}\left(\mathbf{G}^{-1} \mathbf{r}_{k}\right)}{e_{1}^{T} \frac{d}{d t}\left(\mathbf{G}^{-1} \mathbf{r}_{k}\right)}+\epsilon \pi
$$

where $\epsilon=0$ if $e_{1}^{T} \frac{d}{d t}\left(\mathbf{G}^{-1} \mathbf{r}_{k}\right)>0$ and $\epsilon=1$ otherwise. The vector of the new control inputs for this transformed system is $\dot{\psi}=\left(\dot{\psi}_{1}, \ldots, \dot{\psi}_{N}\right)^{T}$.

Applying the circular control law from [4] expressed in the transformed framework, the system (4) converges to $\mathcal{C}_{0}$. Now we want to come back to the original framework to express the control inputs of the original system $v_{k}, u_{k}$ with respect to the transformed control input $\dot{\psi}_{k}$. Considering (1), it is easy to see that $v_{k}$ and $\theta_{k}$ are given by:

$$
v_{k}=\left\|\dot{\mathbf{r}}_{k}\right\| \quad \text { and } \quad \tan \theta_{k}=\frac{\dot{y}_{k}}{\dot{x}_{k}}
$$

An expression of $\dot{\theta}_{k}$ is obtained by computing the derivative of $\tan \theta_{k}$ as follows:

$$
\dot{\theta}_{k}\left(1+\tan ^{2} \theta_{k}\right)=\frac{d}{d t}\left(\frac{\dot{y}_{k}}{\dot{x}_{k}}\right)=\frac{\ddot{y}_{k} \dot{x}_{k}-\dot{y}_{k} \ddot{x}_{k}}{\dot{x}_{k}^{2}}
$$

Using the homogeneous coordinates defined previously, the following equation holds:

$$
\dot{\theta}_{k}=\frac{\ddot{\mathbf{r}}_{k}^{T} \mathbf{R}_{\frac{\pi}{2}} \dot{\mathbf{r}}_{k}}{\left\|\dot{\mathbf{r}}_{k}\right\|^{2}}
$$

The original system is related to the transformed system through the matrix $\mathbf{G}$. The following equations are obtained from the change of coordinates (3):

$$
\begin{aligned}
& \mathbf{r}_{k}=\mathbf{G} \hat{\mathbf{r}}_{k} \\
& \dot{\mathbf{r}}_{k}=\dot{\mathbf{G}} \hat{\mathbf{r}}_{k}+\mathbf{G} \dot{\hat{\mathbf{r}}}_{k}=\mathbf{G G}^{-1} \mathbf{r}_{k}+\omega_{0} \mathbf{G}\left(\cos \psi_{k}, \sin \psi_{k}, 0\right)^{T} \\
& \ddot{\mathbf{r}}_{k}=\ddot{\mathbf{G}} \hat{\mathbf{r}}_{k}+2 \dot{\mathbf{G}} \dot{\hat{\mathbf{r}}}_{k}+\mathbf{G} \ddot{\hat{\mathbf{r}}_{k}}= \\
& =\ddot{\mathbf{G}} \mathbf{G}^{-1} \mathbf{r}_{k}+2 \dot{\mathbf{G}} \dot{\mathbf{G}}^{-1} \mathbf{r}_{k} \\
& +2 \dot{\mathbf{G}} \mathbf{G}^{-1} \dot{\mathbf{r}}_{k}+\mathbf{G R} \frac{\pi}{2}\left(\dot{\mathbf{G}}^{-1} \mathbf{r}_{k}+\mathbf{G}^{-1} \dot{\mathbf{r}}_{k}\right) \dot{\psi}_{k}
\end{aligned}
$$

Thanks to these relations, the original framework is expressed with respect to the transformed system and the circular control law for $\dot{\psi}_{k}$.

\section{B. Formation Control Law}

Using the previous definitions of elastic formation and the general transformation matrix, a new control law is proposed in the following theorem:

Theorem 1 Let $\boldsymbol{G}$ be a twice differentiable matrix with bounded derivatives resulting of a sequence of affine transformations as (2) and $\mathcal{F}=\boldsymbol{G} \circ \mathcal{C}_{0}$ be the desired elastic formation. Let $\omega_{0} \neq 0, \kappa>0$ be two control parameters such that the following condition is satisfied:

$$
\left|\omega_{0}\right| \neq\left\|\boldsymbol{G}^{-1} \dot{\boldsymbol{G}} \boldsymbol{G}^{-1} \boldsymbol{r}_{k}\right\|
$$

Then the control law:

$$
\begin{aligned}
v_{k}= & \left\|\dot{\boldsymbol{G}} \boldsymbol{G}^{-1} \boldsymbol{r}_{k}+\omega_{0} \boldsymbol{G}\left(\cos \psi_{k}, \sin \psi_{k}, 0\right)^{T}\right\| \\
u_{k}= & \frac{1}{v_{k}^{2}}\left(\ddot{\boldsymbol{G}} \boldsymbol{G}^{-1} \boldsymbol{r}_{k}+2 \dot{\boldsymbol{G}} \dot{\boldsymbol{G}}^{-1} \boldsymbol{r}_{k}+2 \dot{\boldsymbol{G}} \boldsymbol{G}^{-1} \dot{\boldsymbol{r}}_{k}\right)^{T} \boldsymbol{R}_{\frac{\pi}{2}} \dot{\boldsymbol{r}}_{k} \\
& +\frac{\dot{\psi}_{k}}{v_{k}^{2}}\left(\dot{\boldsymbol{G}}^{-1} \boldsymbol{r}_{k}+\boldsymbol{G}^{-1} \dot{\boldsymbol{r}}_{k}\right)^{T} \boldsymbol{R}_{\frac{\pi}{2}}^{T} \boldsymbol{G}^{T} \boldsymbol{R}_{\frac{\pi}{2}} \dot{\boldsymbol{r}}_{k}
\end{aligned}
$$

with

$$
\begin{aligned}
\dot{\psi}_{k} & =\omega_{0}\left(1+\kappa \omega_{0}\left(\cos \psi_{k}, \sin \psi_{k}, 0\right) \boldsymbol{G}^{-1} \boldsymbol{r}_{k}\right) \\
\psi_{k}(0) & =\arctan \frac{e_{2}^{T} \frac{d}{d t}\left(\boldsymbol{G}^{-1} \boldsymbol{r}_{k}\right)(0)}{e_{1}^{T} \frac{d}{d t}\left(\boldsymbol{G}^{-1} \boldsymbol{r}_{k}\right)(0)}+\epsilon \pi
\end{aligned}
$$

makes all the agents defined by (1) converge to the elastic formation $\mathcal{F}$. The direction of rotation is determined by the sign of $\omega_{0}$.

Proof: Using the relations between the original system and the transformed system detailed in the previous subsection, starting from (7), the control inputs $v_{k}, u_{k}$ are straightforwardly given by (6). The convergence of the transformed system to the circular formation is analyzed using the following Lyapunov function, based on the analysis of the circular control law proposed in [4]:

$$
S(\hat{\mathbf{r}}, \psi)=\frac{1}{2} \sum_{k=1}^{N}\left\|\dot{\hat{\mathbf{r}}}_{k}-\omega_{0} \overline{\mathbf{R}}_{\frac{\pi}{2}} \hat{\mathbf{r}}_{k}\right\|^{2} \geq 0
$$


where the matrix $\overline{\mathbf{R}}_{\frac{\pi}{2}}$ represents a rotation by $\frac{\pi}{2}$ but erasing the homogeneous coordinate such that $\overline{\mathbf{R}}_{\frac{\pi}{2}}(3,3)=0$. Note that when $S(\hat{\mathbf{r}}, \psi)=0$ the dynamics of the transformed system (4) satisfy $\dot{\hat{\mathbf{r}}}_{k}^{T} \hat{\mathbf{r}}_{k}=0$ which is the kinematic relation for the rotation of the rigid body. Evaluating the derivative of $S(\hat{\mathbf{r}}, \psi)$ along the solutions of the resulting closed-loop system (4) with the control law (7a) leads to:

$$
\begin{array}{r}
\dot{S}(\hat{\mathbf{r}}, \psi)=\sum_{k=1}^{N}\left(\dot{\hat{\mathbf{r}}}_{k}-\omega_{0} \mathbf{R}^{*} \hat{\mathbf{r}}_{k}\right)^{T}\left(\mathbf{R}_{\frac{\pi}{2}} \dot{\hat{\mathbf{r}}}_{k} \dot{\psi}_{k}-\omega_{0} \mathbf{R}_{\frac{\pi}{2}} \dot{\hat{\mathbf{r}}}_{k}\right) \\
=\sum_{k=1}^{N} \omega_{0} \hat{\mathbf{r}}_{k}^{T} \dot{\hat{\mathbf{r}}}_{k}\left(\omega_{0}-\dot{\psi}_{k}\right)=\kappa \sum_{k=1}^{N}\left(\omega_{0} \hat{\mathbf{r}}_{k}^{T} \dot{\mathbf{r}}_{k}\right)^{2} \leq 0
\end{array}
$$

Therefore $S(\hat{\mathbf{r}}, \psi)$ is a suitable Lyapunov function for this transformed system. Thus, the solutions converge to the largest invariant set $\Lambda$, for which $\dot{S}=0$. Then, the transformed system (4) asymptotically reaches the circular formation centered at $(0,0)^{T}$ and of unit radius with fixed angular velocity $\omega_{0}$. Thanks to the change of coordinates (3), the dynamic closed-loop equation corresponding to the transformed formation is time-invariant, hence LaSalle Principle can be applied. As stated above, this result is an adaptation of the circular control law in [4] expressed in the new formulation.

Note that this control law has singular points when $v_{k}=$ 0 , such that:

$$
v_{k}=\left\|\dot{\mathbf{G}} \mathbf{G}^{-1} \mathbf{r}_{k}+\omega_{0} \mathbf{G}\left(\cos \psi_{k}, \sin \psi_{k}, 0\right)^{T}\right\|=0
$$

This singular point occurs if there exists a time $t_{c}$ such that:

$$
\left\{\begin{array}{l}
\left\|\mathbf{G}^{-1}\left(t_{c}\right) \dot{\mathbf{G}}\left(t_{c}\right) \mathbf{G}^{-1}\left(t_{c}\right) \mathbf{r}_{k}\left(t_{c}\right)\right\|=\left|\omega_{0}\right| \\
\angle \mathbf{G}^{-1}\left(t_{c}\right) \dot{\mathbf{G}}\left(t_{c}\right) \mathbf{G}^{-1}\left(t_{c}\right) \mathbf{r}_{k}\left(t_{c}\right)=\psi_{k}\left(t_{c}\right)
\end{array}\right.
$$

where $\angle$ represents the argument of a vector. The equation (5) is a sufficient condition to avoid the singular points.

Remark 1 Note that the equation (5) is a condition imposed to the transformation matrix $\boldsymbol{G}$ to restrict the variation of its time-varying parameters with respect to the angular velocity $\omega_{0}$. In each particular case, it can be expressed in a simple manner and corresponds to an initialization protocol or a physical limitation. For instance, to avoid $v_{k}=0$ in the case of a time-varying translation $\boldsymbol{T}_{c}$, the velocity of the moving circle cannot be equal to the linear velocity of the agents in the circle. The condition (5) becomes $R\left|\omega_{0}\right|>\|\dot{\boldsymbol{c}}\|$ where $R$ is the radius of the circle and $\dot{\boldsymbol{c}}$ the velocity of its center, see [16].

Theorem 1 presents a general control law expressed in the new framework, to stabilize a group of agents to an elastic formation. The term elastic denote the capability of the formation to move and change its shape in order, for instance, to avoid an obstacle (see Fig. 2), to achieve the source seeking problem, to delimit a polluted region, or to avoid unnecessary energy waste. This elastic formation is defined by a sequence of affine transformations $\mathbf{G}$ applied to the unit circle. The matrix $\mathbf{G}$ is a given reference for all the agents. Note that each agent converges to the formation independently of the rest of the fleet. Following section presents a collaborative control to distribute the agents along the formation defined by $\mathbf{G}$.

\section{Cooperative Control: Symmetric Balanced Patterns}

This section is dedicated to the problem of homogenizing the distribution of the agents along the formation. In the unit circle $\mathcal{C}_{0}$, the agents are uniformly distributed when the angular difference between adjacent vehicles is $2 \pi / N$. The distribution of the agents along an elastic formation $\mathcal{F}$ depends on the transformation matrix $\mathbf{G}$ applied to $\mathcal{C}_{0}$.

All-to-all communication topology and some cases of limited communication have been studied in [4], [13] for the circular formation problem. The translation or contraction control laws including this consideration are straightforward obtained, as shown in [16], [17]. The solution proposed in these previous works deals with the addition of a potential function depending on the heading angles of which the minimum corresponds to a symmetric pattern. For instance, the symmetric pattern in which all of the particles are uniformly spaced around the circle is called a splay formation. This potential function contains information of the communication links between the agents. The communication topology for the group of agents is represented by means of a communication graph $\mathcal{G}$. $\mathbf{L}$ denotes the Laplacian matrix of $\mathcal{G}$, see [23].

This paragraph presents the notation included in the corollary. The new Laplacian matrix considered is $\overline{\mathbf{L}}=$ $\mathbf{L} \otimes \mathbf{I}_{2}$ where $\otimes$ is the classical Kronecker product and $\mathbf{I}_{N} \in \mathbb{R}^{N \times N}$ is the identity matrix and the matrix $\mathbf{B}_{m}=\left(\cos m \psi_{1}, \sin m \psi_{1}, \ldots, \cos m \psi_{N}, \sin m \psi_{N}\right)^{T}$ contains all the orientation angles of the transformed system. Considering this notation and applying the previous mentioned works to our new formulation, the following corollary holds:

Corollary 1 Let $\boldsymbol{G}$ be a twice differentiable matrix with bounded derivatives resulting of a sequence of affine transformations as (2) and $\mathcal{F}=\boldsymbol{G} \circ \mathcal{C}_{0}$ be the desired elastic formation. Let $\omega_{0} \neq 0, \kappa>0$ and $K>0$ be three control parameters and the condition (5) be satisfied. Let $\mathcal{G}$ be the communication graph and $\boldsymbol{L}$ be the corresponding Laplacian matrix. Then the previous control law (6) with:

$$
\left\{\begin{array}{l}
\dot{\psi}_{k}=\omega_{0}\left(1+\kappa \omega_{0}\left(\cos \psi_{k}, \sin \psi_{k}, 0\right) \boldsymbol{G}^{-1} \boldsymbol{r}_{k}\right)-\frac{\partial U}{\partial \psi_{k}} \\
U(\psi)=-\frac{K}{N} \sum_{m=1}^{\lfloor N / 2\rfloor} \frac{1}{2 m^{2}} \boldsymbol{B}_{m}^{T} \overline{\boldsymbol{L}} \boldsymbol{B}_{m}
\end{array}\right.
$$

where $\lfloor N / 2\rfloor$ is the largest integer less than or equal to $N / 2$ and the initial conditions $\psi(0)$ satisfy (7b), makes all the agents defined by (1) converge to the formation $\mathcal{F}$. The direction of rotation is determined by the sign of $\omega_{0}$. Moreover, the splay pattern is an extremum point of the potential $U(\psi)$. If the communication graph is complete (allto-all communication) the splay pattern is asymptotically stable and the uniform distribution of the angles $\psi_{k}$ along $\mathcal{C}_{0}$ is achieved. Therefore the agents are distributed in the formation $\mathcal{F}$, taking into account the transformation $\boldsymbol{G}$.

Proof: The stability is analyzed by the composed Lyapunov function $V(\hat{\mathbf{r}}, \psi)=\kappa S(\hat{\mathbf{r}}, \psi)+U(\psi)$ of which the derivative is expressed as $\dot{V}(\hat{\mathbf{r}}, \psi)=\kappa \dot{S}(\hat{\mathbf{r}}, \psi)+\nabla U(\psi)$. 


\begin{tabular}{|l|l|}
\hline Elastic Formation & Transformation \\
\hline Circular formation $((0,0), R=1)$ & $\mathbf{G}=\mathbf{I}$ \\
Circular formation $(\mathbf{c}, R)$ & $\mathbf{G}=\mathbf{T}_{c} \mathbf{S}$ \\
Translation of circular formation & $\mathbf{G}=\mathbf{T}_{c(t)} \mathbf{S}$ \\
Contraction of circular formation & $\mathbf{G}=\mathbf{T}_{c} \mathbf{S}_{R(t)}$ \\
Combined motion & $\mathbf{G}=\mathbf{T}_{c(t)} \mathbf{S}_{R(t)}$ \\
Elliptic formation & $\mathbf{G}=\mathbf{S}_{a, b}$ \\
Rotating elliptic formation & $\mathbf{G}=\mathbf{R}_{\alpha} \mathbf{S}_{a, b}$ \\
\hline
\end{tabular}

TABLE I

ELASTIC FORMATIONS AND THEIR CORRESPONDING TRANSFORMATIONS

Based on the previous works [4], [13], the potential function $U(\psi)$ is invariant to rigid rotations. Therefore, using (8), the derivative of the Lyapunov function satisfies $\dot{V}(\hat{\mathbf{r}}, \psi)=$ $-\sum_{k=1}^{N}\left(\kappa \omega_{0} \hat{\mathbf{r}}_{k}^{T} \dot{\hat{\mathbf{r}}}_{k}-\frac{\partial U}{\partial \psi_{k}}\right)^{2} \leq 0$. Thanks to LaSalle Principle, the system converges asymptotically to the elastic formation and the agents are distributed along $\mathcal{F}$ taking into account the transformation matrix $\mathbf{G}$.

Remark 2 An extension of this result can be proposed for the case of limited communication preserving the same formulation and considering the connectivity properties for the Laplacian matrix which correspond to several communication graphs. See [2], [13], [17], [23].

The cooperative control law (8) is an extension of the previous formation control law to stabilize elastic formations. The splay pattern is an extremum of the potential function $U(\psi)$ which is added to the transformed control variable $\dot{\psi}_{k}$. In the case of limited communication range, a communication area $\rho$ is introduced. This means each agent can only receive information from its close neighbors, i.e if the distance between two agents $k$ and $j$ is smaller than $\rho$, these agents are able to communicate, see [17]. In this case, the function $U(\psi)$ can be also considered as a repulsion potential which is able to avoid the collisions between the agents.

\section{Simulation Results and Particular CAses}

The previous section shows the general control law to stabilize the agents modeled by (1) to a formation defined by the transformation $\mathbf{G}$. The objective of this generalization is to express the previous formation control laws presented in the literature with the new formulation and to propose a solution to control new class of formations. In order to validate this result, some particular cases are presented in this section. First of all, we show that the works on circular formation [4], [13], [16], [17] can be expressed through this formulation. Next, some new formations are proposed. Some exemples are described in Table I.

\section{A. Circular Formation}

The more simple case when $\mathbf{G}=\mathbf{I}_{3}$ is analyzed. The control law becomes:

$$
\begin{aligned}
& v_{k}=\left|\omega_{0}\right| \\
& u_{k}=\dot{\psi}_{k}=\omega_{0}\left(1+\kappa \dot{\mathbf{r}}_{k}^{T} \mathbf{r}_{k}\right)
\end{aligned}
$$

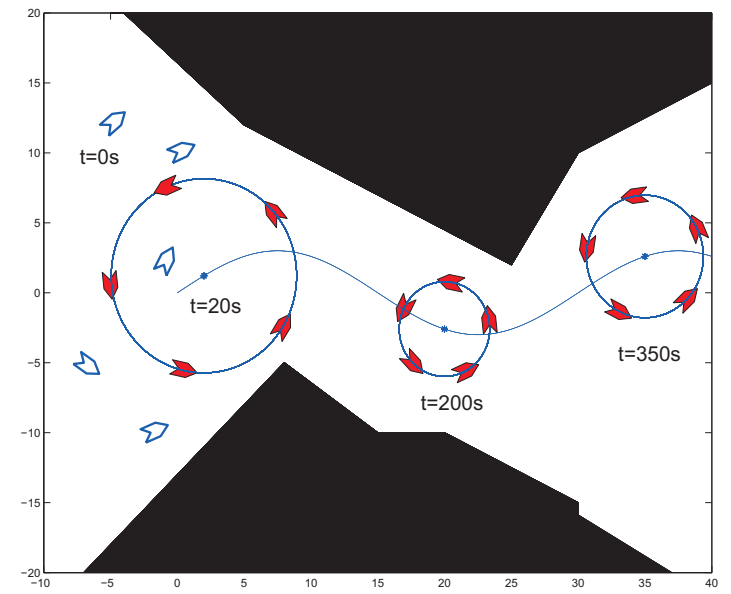

Fig. 2. Simulation of five agents governed by the control law (6) where $\mathbf{G}(t)=\mathbf{S}_{R(t)} \mathbf{T}_{c(t)}$. The circular formation with time-varying radius tracks the time-varying center reference in order to avoid the obstacles (black blocks).

This control law makes all the agents defined by (1) converge to the circular formation $\mathcal{C}_{0}$. This result is equivalent to the circular control law from [4].

In order to stabilize a circular formation with a desired radius $R>0$ and centered at $\mathbf{c}=\left(c_{x}, c_{y}\right)$ the general transformation $\mathbf{G}$ is a sequence of a translation and a uniform scaling $\left(s_{x}=s_{y}=R\right.$ ), such that $\mathbf{G}=\mathbf{T}_{c} \mathbf{S}_{R}$. In this case the control law is expressed as:

$$
\begin{aligned}
& v_{k}=R\left|\omega_{0}\right| \\
& u_{k}=\dot{\psi}_{k}=\omega_{0}\left(1+\kappa \dot{\mathbf{r}}_{k}^{T}\left(\mathbf{r}_{k}-\mathbf{c}^{h}\right)\right)
\end{aligned}
$$

where $\mathbf{c}^{h}$ is the position vector of the center in homogeneous coordinates.

\section{B. Combined Motion of a circle}

The new formulation shown in this article makes possible the combination of several transformations. This is the case of the combined motion problem in which the circular formation with time-varying radius tracks a time-varying center. Consider the transformation $\mathbf{G}(t)=\mathbf{T}_{c(t)} \mathbf{S}_{R(t)}$ which represents a combined motion (translation and scaling) of the circular formation where the center of the desired formation $\mathbf{c}(t): \mathbb{R} \rightarrow \mathbb{R}^{2}$ and its radius $R(t): \mathbb{R} \rightarrow \mathbb{R}^{+}$are twice differentiable functions with bounded first and second time-derivatives. Applying Theorem 1, the agents converge to a circular formation with time-varying radius and moving center. This result is the combination of the two previous works [16], [17] expressed in the new framework.

Figure 2 shows the simulation of five agents governed by the control law (6) with (8) where $\mathbf{G}(t)=\mathbf{T}_{c(t)} \mathbf{S}_{R(t)}$. The control law parameters are $\omega_{0}=1, \kappa=1$ and $K=1 / 10$. The time-varying radius reference is $R(t)=5+2 \cos \frac{2 \pi}{500} t$ and $\mathbf{c}(t)=\left(\frac{1}{10} t, 3 \sin \frac{2 \pi}{300} t\right)^{T}$ is the tracked time-varying center. The agents converge to the moving formation for any random initial conditions (position and heading of the agent) represented in the figure by the blue void agents. This is an 


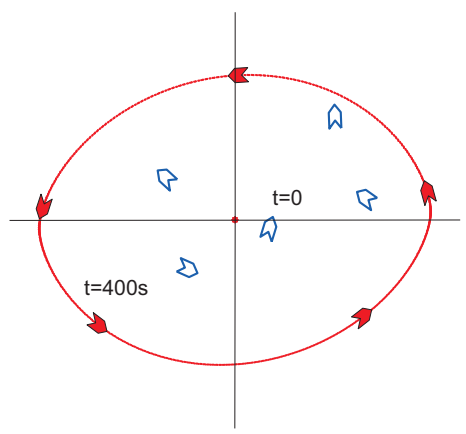

Fig. 3. Simulation of five agents stabilized in an elliptic formation. The red line represents the trajectory of one agent (elliptic formation). The figure shows two snapshots. The blue agents represent the initial conditions. The reds ones represent the final state $t=400 \mathrm{~s}$.

example of one possible application of the combined motion control law and a first step to achieve the final objective: to develop a collaborative control to generate both references in a distributed way.

Moreover, the communication radius considered here is $\rho=10$ which satisfies the geometrical condition $\rho>$ $2 R_{\max } \sin \frac{\pi}{N}$ where $R_{\max }$ is the up-bound of the radius reference. Therefore the agents are distributed along the time-varying circular formation, see [17].

\section{Elliptic formation}

The general formulation presented in this article is pertinent also to stabilize the fleet to non-circular formations as an ellipse. In this case the transformation is a time-invariant non-uniform scaling $\mathbf{G}=\mathbf{S}$ where $s_{x} \neq s_{y}$.

Figure 3 shows a simulation of five agents with the controller designed in Theorem 1 and all-to-all communication. The control law parameters are $\omega_{0}=1, \kappa=1$ and $K=1 / 10$. The agents are stabilized to the elliptic formation defined by the non-uniform scaling $s_{x}=5, s_{y}=$ 1. Moreover the agents are distributed along the formation considering the transformation of the splay pattern which is stable in the original unit circle.

\section{CONCLUSIONS}

We have developed a general control law to stabilize a fleet of agents to an elastic formation, for instance timevarying or non-circular formation, which is defined by a sequence of affine transformations (translation, rotation and scaling) applied to the unit circle. Some particular cases and simulations have been presented to show the convergence of this control law and some possible applications. Moreover, this paper proposes a cooperative control algorithm to distribute the agents along the elastic formation. This potential function is designed by taking into account the communication graph between agents.

At this time, it is assumed that all agents have perfect knowledge of the transformation matrix $\mathbf{G}$ and its first and second derivatives. Further developments would consider a cooperative algorithm which will relax this assumption, and the elastic formation will be defined in a collaborative way.
Moreover, an other future research goal is to include the effect of different disturbances as currents on the formation control algorithm.

\section{REFERENCES}

[1] R. Olfati-Saber, J. A. Fax, and R. M. Murray, "Consensus and cooperation in networked multi-agent systems," Proceedings of the IEEE, 2007.

[2] R. Olfati-Saber and R. M. Murray, "Consensus problems in networks of agents whit switching topology and time-delays," IEEE Transactions on Automatic Control, 2004.

[3] R. Olfati-Saber, "Flocking for multi-agent dynamic systems: Algorithms and theory," IEEE Transactions on Automatic Control, 2006.

[4] N. E. Leonard, D. A. Paley, F. Lekien, R. Sepulchre, D. M. Frantatoni, and R. E. Davis, "Collective motion, sensor networks and ocean sampling," Proceedings of the IEEE, 2007.

[5] P. Ögren, E. Fiorelli, and N. E. Leonard, "Cooperative control of mobile sensor networks: Adaptive gradient climbing in a distributed environment," IEEE Transactions on Automatic Control, 2004.

[6] F. Zhang and N. E. Leonard, "Cooperative filters and control for cooperative exploration," IEEE Transactions on Automatic Control, 2010.

[7] I. Kaminer, A. Pascoal, E. Hallberg, and C. Silvestre, "Trajectory tracking for autonomous vehicles: An integrated approach to guidance and control," Journal of Guidance, Control, and Dynamics, 1998.

[8] E. W. Frew, D. A. Lawrence, and S. Morris, "Coordinated standoff tracking of moving targets using lyapunov guidance vector fields," Journal of Guidance, Control, and Dynamics, 2008.

[9] E. Fiorelli, P. Bhatta, N. E. Leonard, and I. Shulman, "Adaptive sampling using feedback control of an autonomous underwater glider fleet," Proceedings of 13th Int. Symp. on Unmanned Untethered Submersible Technology (UUST), 2003.

[10] R. L. Raffard, C. J. Tomlin, and S. P. Boyd, "Distributed optimization for cooperative agents: Application to formation flight," $43 \mathrm{rd}$ IEEE Conference on Decision and Control, 2004.

[11] S. Martínez, J. Cortés, and F. Bullo, "Motion coordination with distributed information," IEEE Control Systems Magazine, 2007.

[12] P. Ögren, M. Egerstedt, and X. Hu, "A control lyapunov function approach to multiagent coordination," IEEE Transactions on Automatic Control, 2002.

[13] R. Sepulchre, D. A. Paley, and N. E. Leonard, "Stabilization of planar collective motion with limited communication," IEEE Transactions on Automatic Control, 2008.

[14] S. Hernandez and D. A. Paley, "Three-dimensional motion coordination in a time-invariant flowfield," in Proceedings of the 48th IEEE Conference on Decision and Control, 2009.

[15] D. A. Paley and C. Peterson, "Stabilization of collective motion in a time-invariant flowfield," Journal of Guidance, Control, and Dynamics, 2009.

[16] L. Briñón, A. Seuret, and C. Canudas, "Translation control of a fleet circular formation of auvs under finite communication range," in Proceedings of the 48th IEEE Conference on Decision and Control, held jointly with the 28th Chinese Control Conference, 2009.

[17] - "Contraction control of a fleet circular formation of auvs under limited communication range," in Proceedings of the IEEE 2010 American Control Conference, 2010.

[18] - "General framework using affine transformations to formation control design," in Proceedings of the 2nd IFAC Workshop on Distributed Estimation and Control in Networked Systems NECSYS, 2010.

[19] D. A. Paley, N. E. Leonard, and R. Sepulchre, "Stabilization of symmetric formations to motion around convex loops," Systems \& Control Letters, 2008.

[20] B. Moore and C. Canudas, "Source seeking via collaborative measurements by a circular formation of agents," in Prooceedings of the IEEE American Control Conference,, 2010.

[21] N. E. Leonard and E. Fiorelli, "Virtual leaders, artifitial potentials and coordinated control of goups," in Proceedings of 40st IEEE Conf. Decision and Control, 2001.

[22] J. D. Foley, A. van Dam, S. K. Feiner, and J. F. H. andR. L. Phillips, Introduction to Computer Graphics. Addison-Wesley Publishing Co., 1994.

[23] N. Biggs, Algebraic Graph Theory. Cambridge University Press, 1994. 